\title{
The Benefits of Sports Medicine
}

\author{
Dra Mirta D’Ambra* \\ Chairman in Argentina the World Academy Medical Sciences. Specialist Ministry Health, University Buenos Aires, USA
}

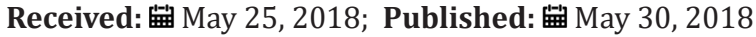

*Corresponding author: Dra Mirta D’Ambra, Chairman in Argentina the World Academy Medical Sciences. Specialist Ministry Health, University Buenos Aires, USA

\section{Opinion}

Physiotherapy and Sports Medicine in a new concept focused not only on rehabilitation or treatment of discomfort or pathologies, but to provide all our experience so that all those who wish to start enjoying sports with health. And there is no doubt that thanks to Sports Medicine we are able to discover our own limits while we can carry out an adequate exercise, which in turn allows us to prevent or improve diseases such as diabetes, hypertension or obesity, among others. Thanks to the different personalized programs of physical exercise, which helps us improve memory, mental function, speed and personal autonomy. Besides, of course, help us improve our own body image, our health in general and to enjoy a better feeling of well-being.

\section{Main benefits of Sports Medicine}
a) Benefits on the metabolism
b) Increase the consumption of fats.
c) Increases oxygen utilization capacity.
d) Reduces cardiac work
e) Improves glucose tolerance.
f) It generates a loss of weight
g) Strengthens the structure of bones.
h) It favors the treatment of diabetes.

i) Collaborate in the maintenance of a full sexual life.

j) Benefits on the heart.

k) Increases circulation in the muscles.

l) It reduces the formation of clots within the arteries.

m) Reduces blood pressure.

n) Benefits on the person from a psychological point of view.

o) Increase self-esteem. p) It reduces stress.

q) Decreases anxiety, anguish and depression.

Sports medicine is the specialty of orthopedics that is responsible for the medical care of athletes, both amateurs and high performance professional athletes. Orthopedists dedicated to this specialty should be familiar with the diagnosis and treatment of the different injuries that an athlete may present, in addition to knowing and attending to situations related to nutrition, the prevention of injuries and the allocation of training programs directed for each athlete. It is important that each evaluation is given an approach according to the age of the athlete, since each individual has different physiological characteristics and at each stage of their life they change and become more specific, especially in older adults and children, who have different abilities and performance than those of a young adult. Each sport has a different pattern of injuries, so the sports doctor must evaluate the patient and base a large part of their evaluation, differential diagnosis and treatment plan on the type of physical activity the patient performs.

It is fundamental in the treatment of an athlete, especially high performance, that there is close communication between the orthopedist and the physical therapist so that personalized work and rehabilitation plans are established in a coordinated manner to return the athlete to their activities safely, without risking a relapse and that it recovers its level and performance as soon as possible. It is necessary to take into account that each person has different physical conditions and that each sport or physical activity has different physical and energetic demands that increase with the passage of time in the same measure in which the athlete improves his performance. This is why sports medicine is necessary; as a specialized doctor can advise the athlete regarding their nutrition, basal conditioning condition, health, training programs, recovery periods and, above all, recommend measures for the prevention of injuries. Although it cannot be ignored that the risk of injury is inherent in the practice of a sport and sometimes injuries are inevitable. Good nutrition is a fundamental element for the success 
of any athlete, because it provides the energy required to train and compete, decreases recovery time, increases strength and development of muscle mass, preserves bone structure strong enough to withstand the wear and tear that exercise implies and increase the speed of recovery from illnesses and injuries.

It is important that an athlete does not skip meals that he stays hydrated before, during and after physical activity and that he consumes high quality proteins. The athlete must avoid periods of prolonged fasting to avoid the depletion of amino acids and maintain their proper hormonal levels and must consume fruits and vegetables rich in minerals to have an adequate adaptation to the stress generated by exercise. The importance of sports medicine today is due to the fact that nowadays it is necessary, even in certain sports regulations, to carry out a medical evaluation prior to the participation of an athlete in any sporting event, this with the purpose of preventing risks for the general health of the athlete. It is important that during the evaluation it is determined if there are medical conditions that could put at risk the integrity of the individual. Therefore, a cardiovascular evaluation is performed on all athletes, since with the exercise the cardiac, pulmonary and vascular demands increase. It is also important to determine the weight of the individual, identify signs of metabolic problems, perform a musculoskeletal evaluation of the entire spine and appendicle skeleton to be able to give an adequate recommendation about the activity and its risks for the athlete, as well identify possible injuries that could be exacerbated by increased physical demands.

Sports medicine plays a very important role here, because on many occasions, due to the demands of the sport business, athletes take their bodies to the limit with the idea of accelerating their recovery process and the only thing they achieve is to worsen or aggravate their injury, it is therefore important that the orthopedist establish an appropriate treatment and make the athlete understand that there is a minimum recovery time that cannot be accelerated, otherwise there is a risk of suffering a relapse or injury worse than the first one. Children and older adults should also exercise regularly and make it a part of their lives, even if it is not done professionally, but it must be taken into account that they have different capacities and their body is in stages of development different from those of an adult. It is important that you do not overdo it or try to do activities in which your body can be affected.

\section{History of Sports Medicine}

This branch of medicine is not a mere subspecialty of orthopaedics and traumatology, its area is so wide that the specialists who practice it must have experience in a wide variety of ailments and areas of the body that are exposed to suffer an ailment, suffering or illness when doing sports. Sports doctors not only cater to professional or high performance athletes, they also take care of the health problems and the diagnosis of injuries and sufferings of amateur athletes or those people who perform physical and sports activities constantly, so they must possessing knowledge not only of traumatology and orthopaedics, but also of nutrition, psychology and sociology, can offer athletes much more complete care than the mere treatment of their injuries, as well as determining when to channel them with another specialist. But sports medicine, as well as sports related injuries, is not a modern and exclusive practice of the 20th century. Its practice extends as far back into the history of mankind as sport itself. The first records of this practice date back to the time of ancient Greece, where the Olympics originated; in these sporting events injuries were even more common than at present.

\section{Conclusion}

As we can see the practice of physical activity is healthy for our body. But never forget the supervision of a sports doctor. Sometimes the consequences can overshadow this bonomia.

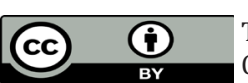

This work is licensed under Creative Commons Attribution 4.0 License

To Submit Your Article Click Here:

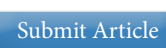

DOI: 10.32474/OSMOAJ.2018.01.000113

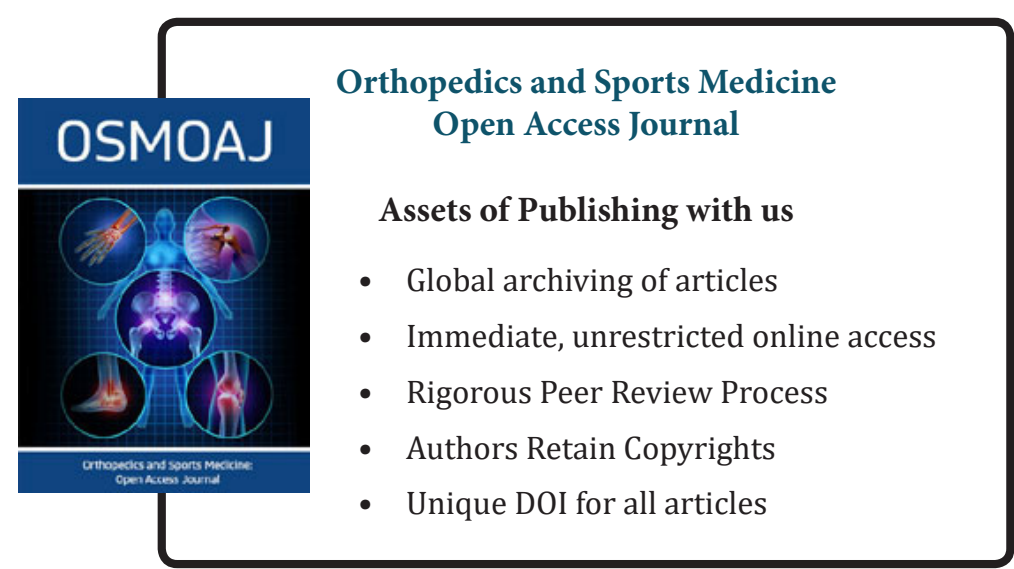

